\title{
1 Consistent marine biogeographic boundaries across the tree of life despite centuries of \\ 2 human impacts
}

3

4

7 Luke E. Holman ${ }^{1, *}($ ORCID: 0000-0002-8139-3760)

8 Mark de Bruyn ${ }^{2,3}$ (ORCID:0000-0003-1528-9604)

9 Simon Creer $^{2}$

10 Gary Carvalho ${ }^{2}$

11 Julie Robidart ${ }^{4}$ (ORCID: 0000-0001-9805-3570)

12 Marc Rius ${ }^{1,5}$ (ORCID: 0000-0002-2195-6605)

$14{ }^{1}$ School of Ocean and Earth Science, National Oceanography Centre Southampton, University

15 of Southampton, United Kingdom

$16{ }^{2}$ Molecular Ecology and Fisheries Genetics Laboratory, School of Natural Sciences, Bangor

17 University, United Kingdom

$18{ }^{3}$ The University of Sydney, School of Life and Environmental Sciences, Australia

$19{ }^{4}$ Ocean Technology and Engineering Group, National Oceanography Centre Southampton,

20 United Kingdom

$21{ }^{5}$ Department of Zoology, Centre for Ecological Genomics and Wildlife Conservation,

22 University of Johannesburg, South Africa

23

24

$25 *$ Corresponding author: lukeearlholman@gmail.com 


\section{Abstract}

28 Over millennia, ecological and evolutionary mechanisms have shaped macroecological

29 distributions across the tree of life. Research describing patterns of regional and global

30 biogeography has traditionally focussed on the study of conspicuous species. Consequently,

31 there is limited understanding of cross-phyla biogeographic structuring, and an escalating need to

32 understand the macroecology of both microscopic and macroscopic organisms. Here we used

33 environmental DNA (eDNA) metabarcoding to explore the biodiversity of marine metazoans,

34 micro-eukaryotes and prokaryotes along an extensive and highly heterogeneous coastline. Our

35 results showed remarkably consistent biogeographic structure across the kingdoms of life, which

36 were underpinned by environmental and anthropogenic influence. Additionally, metazoan

37 communities displayed biographic patterns that suggest regional biotic homogenisation of

38 conspicuous species. Against the backdrop of global pervasive anthropogenic environmental

39 change, our work highlights the importance of considering multiple domains of life to understand

40 the maintenance and drivers of marine biodiversity across broad taxonomic, ecological and

41 geographical scales. 
Introduction

44 Researchers have long recognised the importance of grouping global biota into distinct,

45 geographically separated regions. Delineating these biogeographic areas is important to

46 understand the factors shaping the range limits of species ${ }^{1}$, to designate key geographic areas for

47 biodiversity conservation ${ }^{2}$ and in formulating predictive responses to environmental change $\mathrm{e}^{3,4}$.

48 One of the first efforts to define geographic regions of terrestrial biota were Alfred Russel

49 Wallace's so-called 'Zoological Regions' ${ }^{\text {, }}$, which included six major regions (hereafter realms)

50 that are still recognised in subsequent classifications today ${ }^{6}$. The drivers responsible for these

51 geographic classifications are frequently environmental conditions or physical barriers.

52 Biogeographic studies have shown that deep divergence in the geographic arrangement of

53 terrestrial biota arose as a result of plate tectonics, while shallow divergence has been most

54 frequently attributed to climatic conditions ${ }^{7}$. In aquatic ecosystems, the relative importance of

55 macroecological drivers is less understood, although both climatic (e.g. temperature) ${ }^{8}$ and

56 tectonic forces ${ }^{9}$ have been identified as key determinants of marine biogeographic patterns.

57 Recent studies have successfully partitioned the oceans into distinct ecoregions (i.e. a

58 geographically defined area, smaller than realm, that contains characteristic assemblages of

59 species) $)^{1,10}$, but the description of marine ecoregions has mostly considered conspicuous or well-

60 described species. Similarly, most macroecological marine research has focussed on readily

61 identifiable eukaryotic species, principally metazoans ${ }^{11}$, although demonstrable progress has

62 been made in understanding global patterns of marine microbes ${ }^{12}$. In line with recent studies

63 demonstrating strong cross-phylum interdependence ${ }^{13}$, there is an increasing need to include

64 prokaryotic species in our assessment of biogeographic patterns. The language of macroecology

65 and microbial ecology is similar, both examining the incidence of species across different spatial

66 scales, but these fields have long progressed independently. As a result, relatively few studies

67 have explored biogeographic patterns simultaneously for both microscopic and macroscopic life,

68 with examples of consistent and inconsistent patterns across different taxa ${ }^{13-15}$. Work is thus

69 needed to explore the consistency of biogeographic breaks across different kingdoms of life.

71 Human driven habitat destruction, pollution and the introduction of non-native species are the

72 main drivers of recent global biodiversity change ${ }^{3}$ and therefore have the potential to alter 
geographic patterns of biota at multiple spatial scales. Cumulatively, anthropogenic stressors not

74 only threaten vulnerable native species but whole-community structure and function ${ }^{3,16,17}$. The

75 magnitude and direction of human impacts are complex, with evidence for both gains and losses

76 in local species richness across biomes ${ }^{18-20}$. However, a consistent global pattern is emerging,

77 with a recent and rapid increase in species turnover ${ }^{19}$ and an associated increase in community

78 similarity ( $\beta$ diversity) between two or more geographically separated sites ${ }^{20}$. Incidences of

79 increased community similarity are known as biotic homogenisation ${ }^{21}$ and are driven by human

80 activities that promote extinctions and the introductions of non-native species. In light of

81 growing evidence that taxonomic, phylogenetic and functional diversity are strongly correlated ${ }^{22}$,

82 the homogenisation of biological communities has the potential to negatively affect ecosystem

83 function. Furthermore, even uncommon species within an ecological community can contribute

84 significantly to ecosystem function ${ }^{23}$, demonstrating the importance of studying inconspicuous

85 species to preserve ecosystem health. Studies have shown evidence for biotic homogenisation

86 around the globe, with examples from plants ${ }^{18}$, vertebrates ${ }^{24}$ and invertebrates ${ }^{25}$ demonstrating

87 alteration of terrestrial biogeographic patterns. However, many studies are of limited taxonomic

88 scope, focussing on highly conspicuous species for which reliable data can be relatively easily

89 produced $^{24,25}$. Thus, most work overlooks inconspicuous species (e.g. microbes and microscopic

90 eukaryotes), which show vastly different replication, demographic and dispersal patterns

91 compared to metazoans ${ }^{13}$, but are known to be key actors shaping the assembly of ecological

92 communities and ultimately underpin ecosystem functioning ${ }^{26}$. Taken together, a more

93 comprehensive characterisation of ecological communities is clearly needed when testing the

94 role of biotic homogenisation on biogeographic patterns.

96 The advent of high-throughput sequencing has revolutionised our understanding of microbial

97 life, with studies examining global patterns of prokaryotic life now increasingly common ${ }^{12}$.

98 Moreover, the recent and rapid development of methods to infer the incidence of larger

99 organisms using genetic material isolated from environmental samples (known as environmental

100 DNA or eDNA) has provided an unparalleled ability to identify species across the entire tree of

101 life ${ }^{14,15,27}$. Together, these methods can rapidly generate standardised biodiversity data for entire

102 communities at an unprecedented resolution, thereby minimising regional and taxonomic biases.

103 Such studies provide datasets that can be analysed without taxonomic assignment and DNA 
104 samples can be repurposed to test novel hypotheses. A common technique is to amplify DNA

105 barcodes from eDNA and use high-throughput sequencing to produce high-resolution

106 biodiversity data. This method (eDNA metabarcoding) has been shown to reliably detect

107 organisms across many different ecosystems ${ }^{27}$, but has infrequently been applied to understand

108 spatial patterns of biodiversity across different kingdoms of life $\mathrm{e}^{12,15}$.

109

110 A unique geographic setting for testing biogeographic hypotheses is the South African coastline,

111 where two large water masses (the Atlantic and Indian Oceans) meet, and a wide variety of

112 abiotic and biotic conditions are found in a relatively small region. This coastline has three well-

113 defined coastal ecoregions bounded by the cold western boundary Benguela Current and the

114 warm oligotrophic eastern boundary Agulhas Current. These ecoregions have been established

115 on the basis of studies over several decades involving a number of conspicuous metazoan

$116 \operatorname{taxa}^{2,28}$. Additionally, there is evidence for human exploitation of marine resources in the region

117 spanning thousands of years ${ }^{29,30}$ and some areas of the coastline have been subject to heavy

118 maritime activity for centuries ${ }^{31}$. Other human activities also prevail such as the establishment of

119 aquaculture facilities or the construction of harbours and breakwaters ${ }^{29,30}$. Thus, the South

120 African coastline is an ideal study system to explore the mechanisms shaping biogeographic

121 boundaries.

122

123 Here we used eDNA metabarcoding to compare the biogeography of multiple marine kingdoms

124 along the diverse coastline of South Africa and to test the effects of life-history strategies and

125 anthropogenic and natural pressures on biogeographic patterns. We first investigated the

126 consistency of biogeographic boundaries across metazoans, protists and bacteria, and to what

127 extent these patterns could be explained by environmental conditions. We then evaluated if there

128 was evidence for homogenisation of ecological communities along a coastline that has been

129 affected by centuries of human impacts, and to what extent these patterns persisted across

130 taxonomic groups.

\section{Methods}




\section{Field sampling}

136 We sampled a range of sites along 2,000 km of coastline (Fig. 1) between October and

137 November of 2017 (see details in Supplementary Table 1), covering the three major marine

138 ecoregions of South Africa. In order to assess the effects of anthropogenic impacts, we compared

139 human altered 'artificial' sites (e.g. recreational marinas, harbours) and relatively unaltered rocky

140 shore and natural harbour sites, hereafter 'natural' (see Supplementary Table 1). The artificial

141 sites were previously sampled in Rius, et al. ${ }^{32}$, and six adjacent natural sites were selected. The

142 natural sites were the nearest non-developed sites with matching aspect and exposure (Fig. 1) to

143 each of the artificial sampling sites. Three $400 \mathrm{ml}$ seawater samples were filtered with $0.22 \mu \mathrm{m}$

144 polyethersulfone membrane Sterivex filters (Merck Millipore, MA, USA) following the

145 sampling scheme of Holman, et al. ${ }^{33}$ at each sampling site. Consequently, we sampled a total of

$1461,200 \mathrm{ml}$ of seawater per site, a volume that has been shown to differentiate fine scale $\left(<1 \mathrm{~km}^{2}\right)$

147 community structure in marine systems ${ }^{33,34}$. Filters were immediately preserved at ambient

148 temperature with the addition of $1.5 \mathrm{ml}$ of Longmire's Solution for preservation until DNA

149 extraction. Field control filters and equipment cleaning blanks were taken, transported, stored

150 and sequenced as the rest of the field samples.

Environmental DNA extraction

153 We used a PCR-free laboratory separated from the main molecular biology laboratory facilities.

154 No post-PCR or high concentration DNA samples were permitted in the laboratory. All surfaces

155 and lab equipment were cleaned thoroughly before use with 1.25\% sodium hypochlorite solution

156 (3:1 dilution of household bleach). DNA extraction followed the SXCAPSULE method from Spens,

157 et al. ${ }^{35}$. Briefly, filters were first externally cleaned with sterile water and Longmire's Solution

158 was removed from the filter outlet using a sterile syringe, $720 \mu 1$ Buffer ATL (Qiagen, Hilden,

159 Germany) and $80 \mu \mathrm{l}$ Proteinase K $(20 \mathrm{mg} / \mathrm{ml})$ was added and filters were incubated overnight at

$16056^{\circ} \mathrm{C}$. The lysate was then removed from the filter inlet and subjected to DNA extraction using

161 the Qiagen DNeasy Blood and Tissue Kit under the manufacturers recommended protocol. DNA

162 was eluted using $200 \mu \mathrm{l}$ Qiagen Buffer AE and re-eluted once to increase DNA yield. All DNA

163 samples were checked for PCR inhibition using the Primer Design Internal Positive Control

164 qPCR Kit (Primer Design, Southampton, UK) with $10 \mu 1$ reactions under the manufacturer

165 recommended protocol. Inhibition was detected by an increase of $>1.0 \mathrm{Ct}$ in reactions containing 
eDNA compared to reactions with extraction controls. As inhibition was detected in a minority of samples, all samples were treated using the Zymo OneStep PCR Inhibition Removal Kit (Zymo Research, California, USA) following the manufacturer recommended protocol. Inhibited samples showed no evidence for inhibition post cleaning.

\section{High throughput eDNA amplicon sequencing}

172 Different sets of primers were used to generate three separate eDNA metabarcoding libraries for

173 all samples. Two gene regions were selected to target broad metazoan/eukaryotic diversity, a

174 section of the V4 region of the nuclear small subunit ribosomal DNA (hereafter $18 \mathrm{~S})^{36}$ and a

175 section of the standard DNA barcoding region of cytochrome c oxidase subunit I (hereafter

$176 \mathrm{COI})^{37}$. One gene region was used to target prokaryotes, the V3-V4 hypervariable region of 177 prokaryotic small subunit ribosomal DNA (hereafter 16S) ${ }^{38}$. Illumina unique double-indexed

178 metabarcoding amplicon libraries were constructed with a two-step PCR protocol as detailed in

179 Holman, et al. ${ }^{33}$. The first PCR setup was performed in a PCR-free laboratory. The three eDNA

180 samples per site were pooled and three independent technical replicates were sequenced per pool.

181 The process per sequenced pool was as follows. The first PCR reaction was conducted in

182 triplicate in a total reaction volume of $20 \mu \mathrm{l}$. Each reaction contained $10 \mu \mathrm{l}$ Amplitaq GOLD 360

183 2X Mastermix (Applied Biosystems, California, USA), $0.8 \mu \mathrm{l}\left(5 \mathrm{nmol} \mathrm{ml}^{-1}\right)$ of each forward and

184 reverse primers and $2 \mu \mathrm{l}$ of undiluted environmental DNA template. The reaction conditions for

185 PCR were an initial denaturation step at $95^{\circ} \mathrm{C}$ for 10 minutes followed by 20 cycles of $95^{\circ} \mathrm{C}$ for

18630 seconds, variable annealing temp $\left(46^{\circ} \mathrm{C}\right.$ for $\mathrm{COI}, 50^{\circ} \mathrm{C}$ for $18 \mathrm{~S}$ and $55^{\circ} \mathrm{C}$ for $\left.16 \mathrm{~S}\right)$ for 30

187 seconds, and extension at $72^{\circ} \mathrm{C}$ for 1 minute. A final extension at $72^{\circ} \mathrm{C}$ was performed for 10

188 minutes. The triplicate first PCR replicates were then pooled and cleaned using AMPure XP

189 beads (Beckman Coulter, California, USA) at 0.8 beads:sample volume ratio following

190 manufacturer's instructions. The second PCR reaction was conducted in a total volume of $20 \mu 1$

191 containing $10 \mu \mathrm{l}$ Amplitaq GOLD 360 2X Mastermix, $0.5 \mu \mathrm{l}\left(10 \mathrm{nmol} \mathrm{ml}^{-1}\right)$ of both forward and

192 reverse primers and $5 \mu \mathrm{l}$ of undiluted cleaned PCR product from the first reaction. PCR

193 conditions were an initial denaturation step at $95^{\circ} \mathrm{C}$ for 10 minutes followed by 15 cycles of

$19495^{\circ} \mathrm{C}$ for 30 seconds, annealing at $55^{\circ} \mathrm{C}$ for 30 seconds, and extension at $72^{\circ} \mathrm{C}$ for 1 minute. A

195 final extension at $72^{\circ} \mathrm{C}$ was performed for 10 minutes. PCR 2 products were cleaned using

196 AMPure XP beads as above. Negative control samples for the filters, extraction kit, PCR1 and 2 
197 were included in library building and sequenced alongside experimental samples. Products were

198 quantified following the manufacturer's instructions using the NEBNext Library Quant qPCR kit

199 (New England Biolabs, Massachusetts, USA) and then normalised and pooled at an equimolar

200 concentration for each marker. Each gene region was sequenced independently using a V3

201 paired-end 300bp reagent kit on the Illumina MiSeq Instrument with 5\% PhiX genomic library

202 added to increase sequence diversity.

\section{Bioinformatics}

205 Raw sequences were de-multiplexed using the GenerateFastQ (v2.0.0.9) module on the MiSeq 206 control software (v3.0.0.105). Cutadapt (v2.3) ${ }^{39}$ was used to filter sequences to include only 207 those that contained both the forward and reverse primer sequence across both read pairs for 208 each gene fragment, remaining sequences then had the primer region removed for each gene 209 fragment using the default settings. Sequences were denoised using the DADA2 pipeline $210 \quad(\mathrm{v} 1.12)^{40}$ in $\mathrm{R}(\mathrm{v} 3.6 .1)^{41}$ with the default parameters unless noted as follows. Sequences were

211 filtered to retain only pairs of reads with an expected error of 1 or fewer per read. Read trimming

212 was performed after manual examination of the read quality profile, the forward reads were

213 trimmed to $250 \mathrm{bp}(\mathrm{COI}), 240 \mathrm{bp}(18 \mathrm{~S})$ and $240 \mathrm{bp}(16 \mathrm{~S})$ and the reverse reads were trimmed to

$214230 \mathrm{bp}(\mathrm{COI}), 220 \mathrm{bp}$ (18S) and $220 \mathrm{bp}(16 \mathrm{~S})$. As each marker was sequenced separately, the

215 differences in read trimming length reflect typical variation in sequencing runs rather than any

216 biological difference. The error rates per run were estimated and used to perform the denoising

217 using the DADA2 algorithm. The denoised sequence pairs were then merged and resulting

218 sequences were truncated if they were outside of the expected gene fragment range (303-323bp

219 for COI, 400-450bp for 18S and 390-450bp for 16S). Chimeras were identified and removed

220 before assembling a sample by ASV (amplicon sequence variant) table for analysis. The

221 denoised ASVs were then curated using the default settings of the LULU algorithm ${ }^{42}$ which

222 merges sequences based on sequence similarity and co-occurrence. Assigning taxonomy to a set

223 of unknown sequences is a difficult task, particularly considering many marine species lack

224 DNA barcodes, are undescribed, or have erroneous barcodes in online public databases. We

225 therefore focused our analysis at a higher taxonomic level than species, assigning taxonomy to

226 sequences from the COI and $18 \mathrm{~S}$ data as follows. The RDP classifier (v2.13) ${ }^{43}$ was used to

227 assign taxonomy for COI using a previously published COI database ${ }^{44}$ (v4.0) and a modified 
version of the SILVA database ${ }^{45}$ (v3.2 from https://github.com/terrimporter/18SClassifier). As species level assignments have been shown to be accurate for COI data ${ }^{33}$ an unconstrained (no limits on sequence similarity or match length) BLAST search (v2.6.0+) was performed for each

231 sequence against the entire National Centre for Biotechnology Information nt database

232 (downloaded on 16 ${ }^{\text {th }}$ May 2019), 200 hits per sequence were retained (-num_alignments). These

233 sequences were then parsed using an $\mathrm{R}$ script to exclude hits below $65 \%$ coverage, remaining

234 assignments with percent identity above 97\% for COI were used to collapse reads for ASVs

235 assigned to the same species. Recent analyses have suggested that only exact (100\% identity)

236 matching of sequences to reference data is appropriate for species assignment for the prokaryotic

$23716 \mathrm{~S}$ region ${ }^{46}$. The $16 \mathrm{~S}$ sequences were matched to the SILVA database (release 132$)^{45}$ using the

238 default settings of the assignTaxonomy function from the DADA2 package to assign taxonomy

239 at genus level or above. The incidence of NUMTs (nuclear mitochondrial DNA) and chimeras in

240 the final ASV list was evaluated following Supplementary Information 1.

242 The following quality control filters were applied to the ASV by sample table produced by 243 DADA2. First, the minimum number of reads per observation was set at three. Any ASVs not 244 represented in at least one other sample were discarded. ASVs were then filtered to retain only 245 those found in all three technical replicates. For any ASV found in the negative control samples,

246 the largest value among the control samples was used as the zero value for all other samples (i.e.

247 any smaller values found in non-control samples were set to zero). The COI and 18S datasets

248 were then subset by the RDP classifier taxonomic assignments to produce datasets for the 249 protists and metazoans as follows. Phylum level assignments above a threshold of 30, a value 250 well above that shown to accurately assign phylum level taxonomy ${ }^{44}$, were parsed to include 251 phyla that contained only metazoan or protist members for each group respectively, other

252 assignments or unknown assignments were discarded. This resulted in a protist and metazoan 253 dataset for each marker, which were subsequently used for separate analyses using these 254 groupings. The 16S data was parsed to include only bacterial ASVs. Within each taxonomic 255 dataset samples were then rarefied to the smallest number of reads (see Supplementary Table 2). 256 Technical replicates were then collapsed to produce a dataset containing the mean value of 257 rarefied reads per ASV. Finally, ASVs assigned using BLAST (with no cases of multiple 258 matches of equal quality) to the same species in the COI dataset were combined by summing 
reads per site. The taxonomic assignment method used for the $16 \mathrm{~S}$ data assigns to genus, and species level assignments are not possible using the selected $18 \mathrm{~S}$ region, so no ASVs from these datasets were collapsed. In order to explore broad scale patterns of taxonomic diversity, the number of ASVs per phyla and number of rarefied reads per phyla were collapsed to produce per site assessments of taxonomic composition. As phylum level phylogeny is not resolved for all protist species, the protist dataset was grouped by supergroup designations according to Burki, et al. ${ }^{47}$. For plots, phyla represented by less than $2 \%$ of ASV counts were concatenated in an 'other' category.

\section{Abiotic, human impact and geographic data}

269 In situ temperature data reflects a snapshot of the total conditions experienced across the lifetime

270 of the species that make up marine communities. Therefore, abiotic variables for the sites

271 covering an ecologically relevant timescale were sourced as follows. High resolution $\left(1 \mathrm{~km}^{2}\right)$

272 remote sensing average daily sea surface temperature (SST) data derived from multiple satellite 273 missions, combined with in situ data ${ }^{48}$ was parsed in $\mathrm{R}$ to find the nearest datapoint to each site.

274 For each point, a mean from two years of data from November 2017 was calculated. Interpolated 275 average (2005-2017) sea surface salinity (SSS) data $\left(0.25^{\circ}\right.$ grid resolution) generated using 276 gliders, oceanographic casts etc. from the 2018 World Ocean Atlas ${ }^{49}$ was parsed to include only

277 surface data for the sites. Monthly global ocean colour data $\left(4 \mathrm{~km}^{2}\right)$ derived from multiple

278 satellite missions ${ }^{50}$ was parsed to calculate an average value for chlorophyll $a$ density per site

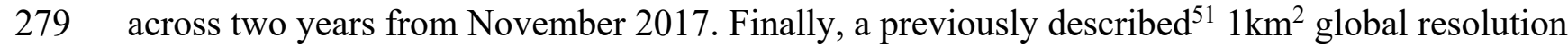

280 cumulative index for anthropogenic impact on marine ecosystems, comprising fishing pressure, 281 climate change, shipping and land-based pollution, was parsed to produce a value for each site 282 cumulatively across the entire period for which data were available (2003-2013). These global

283 datasets have excellent temporal resolution, but are only appropriate for testing large-scale 284 patterns as they have limited ability to discriminate highly localised observations.

Ecological statistics

287 Differences in the mean number of ASVs per coastline were assessed using an analysis of 288 variance (ANOVA) after testing for normally distributed residuals using a Shapiro-Wilk test and 289 equal variance between coasts using a Bartlett test. A Tukey’s Honest Significant difference test 
290 was used to evaluate significant ANOVA results. Differences in community similarity were

291 assessed using a Permutational Multivariate Analysis of Variance (PERMANOVA) ${ }^{52}$

292 implemented in $\mathrm{R}$ with the function adonis from the package vegan (v2.5-6) ${ }^{53}$ to assess

293 differences in multivariate centroids and dispersion between coastlines. The PERMANOVA was

294 conducted on a matrix of Jaccard dissimilarities as this ecological index has been shown to be

295 appropriate for biogeographical studies ${ }^{54}$. Significant pairwise differences were assessed using

296 the R function adonis.pair from the EcolUtils package (v0.1) ${ }^{55}$. To analyse if groups of samples

297 have a difference in intra-group community variation, also known as heterogeneity of

298 multivariate dispersion, the PERMDISP2 procedure ${ }^{56}$ was used, implemented in the R function

299 betadisper from the vegan package. The pairwise group differences in heterogeneity of

300 multivariate dispersion in the case of a global significant result from betadisper were analysed

301 using a Tukey's Honest Significant difference test. Non-metric multidimensional scaling

302 ordinations (nMDS) were calculated using Jaccard dissimilarities and the R function metaMDS

303 from the vegan package.

305 The influence of the abiotic and human impact data on the observed patterns of beta diversity

306 were evaluated as follows. It has previously been common to use a partial Mantel test to evaluate

307 the effect of a distance matrix (frequently environmental variables) on a second distance matrix

308 (species composition) while 'cancelling out' the effect of a third matrix (geographic distance).

309 However, this approach has been shown to be sensitive to spatial autocorrelation common in

310 ecological datasets ${ }^{57}$. A recently developed method ${ }^{57}$, which corrects spurious inflations of the

311 parameter estimate for Mantel tests, was implemented in R. Across each taxonomic group

312 Mantel tests were conducted comparing Jaccard dissimilarity against Euclidean distance for each

313 environmental variable. For each test Moran spectral randomisation was performed including the

314 geographic distance data with 10,000 permutations to assess statistical significance using the $m s r$

315 function in the adespatial package (v0.3-8).

317 Explanatory variables which had some correlation with the community dissimilarity after

318 adjusting for geographic distance were then evaluated as follows. First, a distance-based

319 redundancy analysis $(\mathrm{dbRDA})^{58}$, regressing site Jaccard dissimilarities against all remaining

320 variables, was performed using the $\mathrm{R}$ function $d b r d a$ from the vegan package. The significance 
321 of terms was assessed with 10,000 permutations. The dbRDA ordination allows us to examine

322 linear changes in the beta diversity in response to a number of predictor variables in tandem, and

323 also to explore their relative impact. The function varpart from the vegan package was then used

324 to partition the variance in the community dissimilarity by the environmental variables. We then

325 used a generalised additive model to visualise the variation of each significant variable across the

326 nMDS space via a restricted maximum likelihood 2D smoother, implemented in the function

327 ordisurf in the R package vegan.

329 Distance-decay relationships were explored by first measuring compositional similarity (1-

330 Jaccard index) for each pair of sites, and then calculating distances between pairs of sites by

331 drawing a continuous transect $1 \mathrm{~km}$ offshore parallel to the high-water mark using Google Earth

332 Pro (v 7.3.2.5776), taking the distance along the transect to measure distance between sites. We

333 then used these data in least-square regression models using the $\mathrm{R}$ function $l m$ with an interaction

334 function between distance and site type (artificial or natural) terms against compositional

335 similarity as a response term. The compositional similarity values were $\log 10$ transformed to

336 linearise the response, untransformed values of zero (no overlap of species) were omitted to

337 avoid infinite response variable values.

\section{Results}

\section{Sequencing}

343 A total of 66.25 million sequences were produced across the three sequencing runs. The number

344 of unfiltered raw reads per experimental sample ranged from 61,958 to 859,580 , with an average

345 per sample across all three markers of 347,536 \pm 109,665 (s.d.) (see Supplementary Table 2 for

346 further details). Negative control samples exhibited very low levels of cross-contamination

347 (Supplementary Information 2).

349 Taxonomic assignments \& alpha diversity

350 Analyses for each taxonomic group are presented for the marker which had the largest number of 351 observations (COI for metazoans, 18S for protists), analyses for the remaining subsets are 
352 presented in Supplementary Information and were consistent with the main results. After

353 taxonomic assignment to phyla 1,054, 1,433 and 2,826 ASVs were retained for the metazoans,

354 protist and bacteria datasets respectively. Across all taxonomically grouped datasets the majority

355 of detected ASVs came from a small number $(<5)$ of phyla or supergroups (Figure 1b). This

356 pattern was consistent across sites and no major changes in the identity of ASVs at phyla or

357 supergroup level across the study region were detected (Figure 1b).

359 Across all markers the greatest mean ASV richness was found along the southern coast (Fig. 1).

360 However, a one-way ANOVA showed a significant difference $\left(F_{2,15}=7.18, p=0.007\right)$ between

361 coastlines only in the bacterial dataset with no difference found in the metazoan $\left(\mathrm{F}_{2,15}=1.941\right.$,

$362 \mathrm{p}=0.178)$ or protist data $\left(\mathrm{F}_{2,15}=1.416, \mathrm{p}=0.273\right)$. A post-hoc Tukey test of the bacterial data

363 (Supplementary Table 3) showed that the east and west coasts had significantly fewer ASVs

364 compared to the south coast, but that they were not significantly different to one another in

365 overall ASV richness. 


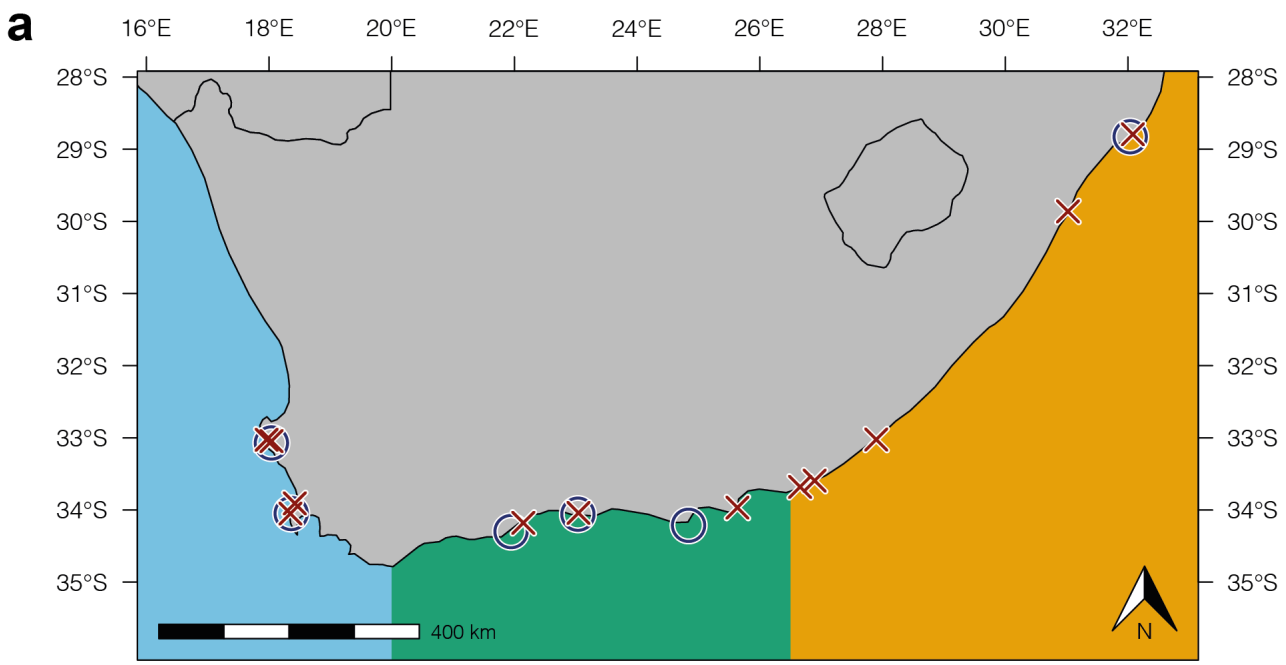

SY SN SM TB HB HN MB MN KN NK CN PE BR PA EL DU RB RN $X 0 \times \times \times O \times O O O \times \times \times \times \times \times 0$

$\mathbf{b}_{\mathbf{i}}$

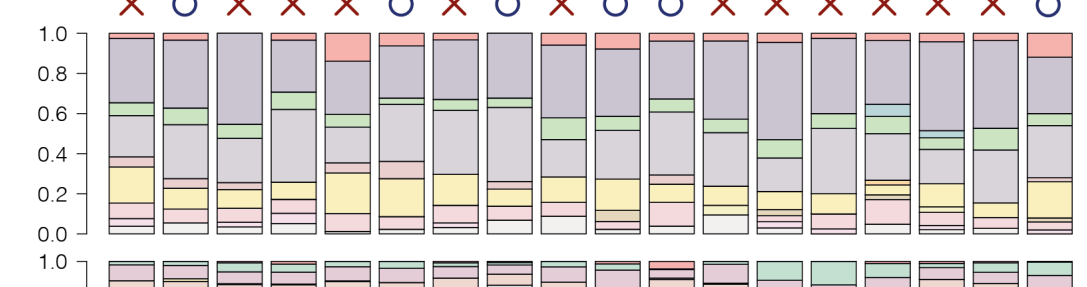

ii
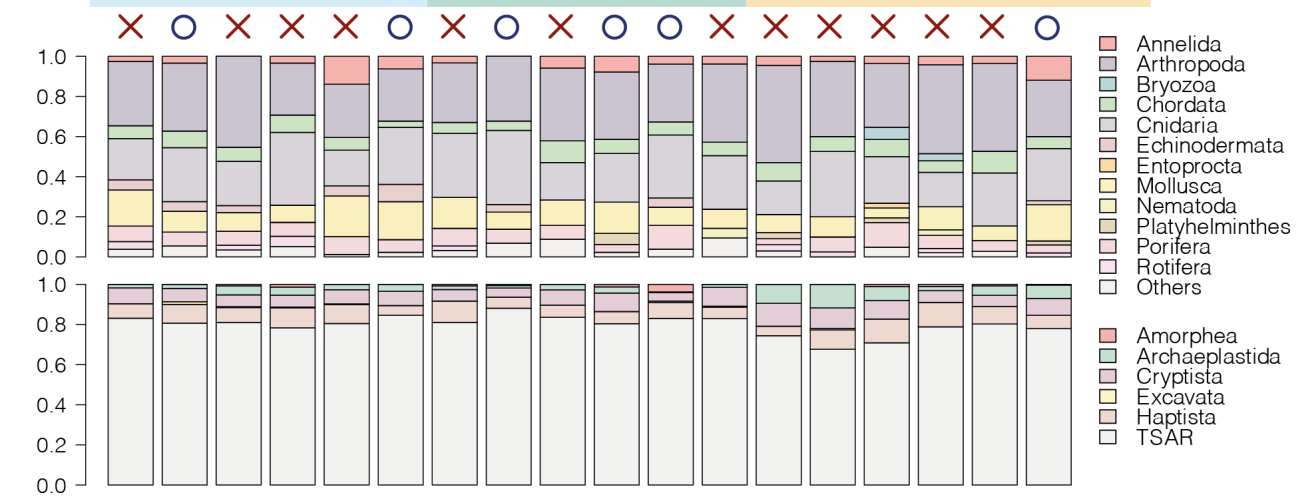

iii
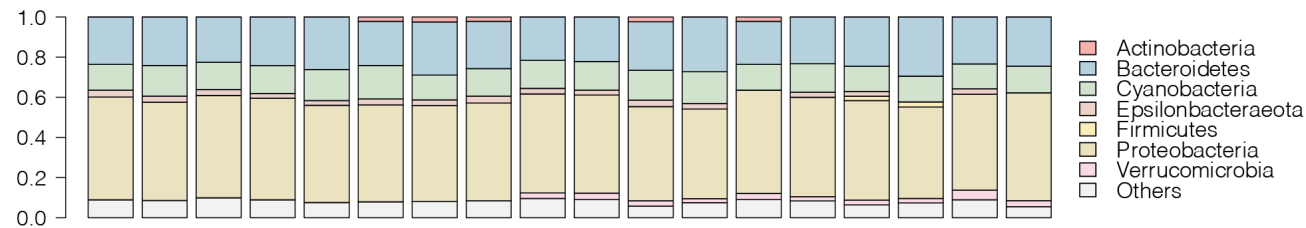

C
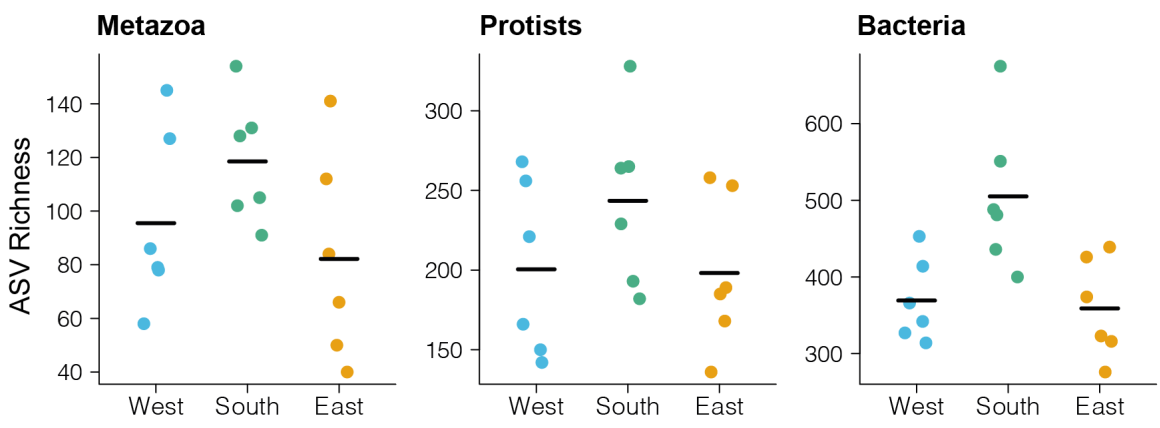

Figure 1. a Map of South Africa indicating the sampling sites and the site types (red crosses are artificial sites and blue circles natural sites). Site codes as in Supplementary Table 1; b Proportion of ASVs per phyla across each site for $\mathbf{i}$ metazoans, ii protists and iii bacteria. Each bar represents a site indicated by by coast and taxonomic group, black line indicates mean ASV richness. 
Beta diversity

376 Across all three taxonomic groups, non-metric multidimensional ordinations showed clustering

377 of sites consistent with ecoregions previously described in conspicuous metazoan species (Fig.

378 2). Furthermore, PERMANOVA models showed a significant $(\mathrm{p}<0.001)$ effect of coastline in all

379 cases (see Supplementary Table 4 for model output), with pairwise significant differences

$380(\mathrm{p}<0.01)$ between all pairs of coastlines in all taxa (Supplementary Table 5). There was evidence

381 (ANOVA on betadispr $\mathrm{F}_{2,15}=4.09, \mathrm{p}=0.038$ ) for heterogeneity of multivariate dispersion in the

382 bacterial dataset (Supplementary Table 6). A Tukey test revealed a significant $(\mathrm{p}=0.031)$

383 pairwise difference between the east and west coast only, in line with the observations of Fig. 2.,

384 indicating that for bacteria, sites on the west coast were more variable in community composition

385 than the more homogenous communities found at sites on the east coast. When the datasets were

386 divided by phylum, they demonstrated consistent evidence for the same significant difference

387 between ecoregions across all tested phylum $(p<0.05$ in all cases, see Supplementary Information

3883 for full model output and visualisation). A power analysis (Supplementary Information 4)

389 indicated that the number of ASVs allocated to each phylum was sufficient to detect a significant

390 difference given the study design.

392 The corrected Mantel tests indicated that in the metazoan and bacterial datasets, SST and the 393 human impact index were significantly correlated with the observed ASV dissimilarities after 394 geographic distance between sites was accounted for (SST p $<0.05$ in all cases; human impact $395 \mathrm{p}<0.01$ in all cases; full model outputs shown in Supplementary Table 7). In the protist dataset 396 chlorophyll $a$ concentration and the impact index remained significant ( $>0.05$ in both cases). In 397 contrast, SSS showed no correlation ( $p>0.05$ in all cases) with observed ASV dissimilarities in 398 any marker. These results indicate that both geographic and environmental distance have some 399 effect on the observed community structure and confirmed that the appropriate variables were 400 retained for analysis in each dataset. In all cases, partial Mantel tests gave similar R statistics and 401 p values (Supplementary Table 7).

403 The dbRDA showed a significant effect of both environmental variables $(\mathrm{p}<0.001$ for SST and 404 chlorophyll $a$ in all cases) and human impact ( $<<0.05$ in all cases) on the site similarity in 
405 metazoans, protists and bacteria, full model outputs are presented in Supplementary Table 8.

406 Variance partitioning of the dbRDA models showed that human impact had a relatively smaller

407 contribution to the observed dissimilarities compared to the chlorophyll $a$ concentration or sea

408 surface temperature as shown in Figure 2. Across taxonomic groups there was negligible overlap

409 in the variance explained by impact and chlorophyll or temperature. Generalised additive models

410 with a 2D smoothed function showed significant terms ( $>0.001)$ across all models (individual

411 full model outputs shown in Supplementary Table 9), indicating that each variable separately

412 explains variation in the eDNA data between communities. SST and chlorophyll $a$ concentration

413 showed surfaces across nMDS plots for all markers (Fig. 2) that were simple, with gradients that

414 were consistent with ecoregions. In contrast, human impact scores showed more complex

415 surfaces with multiple peaks across the ecoregions. 

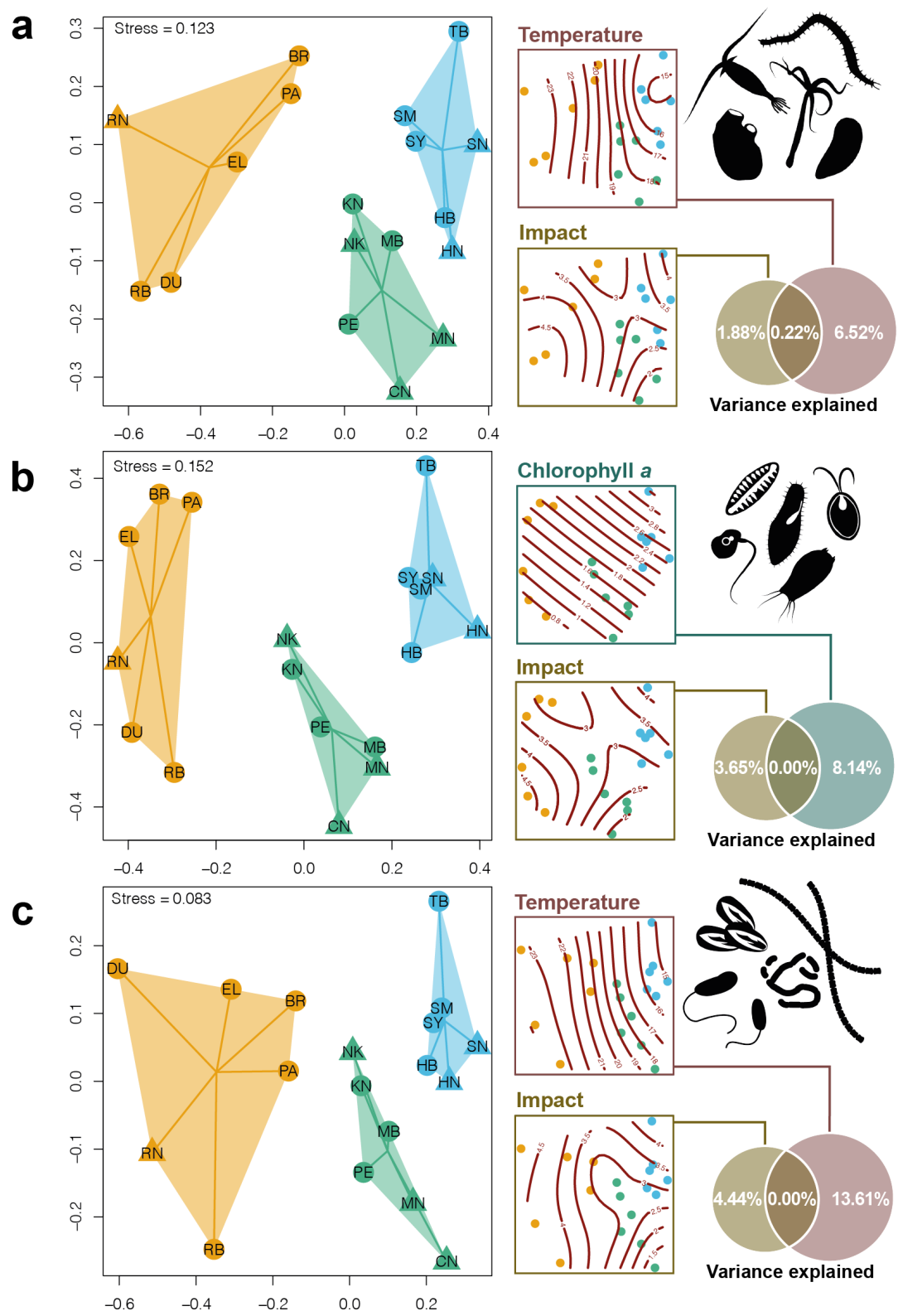

Figure 2. Observed patterns of $\beta$-diversity from environmental DNA metabarcoding of: a metazoans, b protists and $\mathbf{c}$ bacteria; based on Jaccard dissimilarities between amplicon sequence variants along the coast of South Africa. The first column of plots shows non-metric multidimensional scaling (nMDS) ordinations. Coloured hulls show the spread of the data and lines indicate the spread around the centroid grouped by coast with the east, south and west coasts denoted by orange, green and blue respectively. Site abbreviations correspond with Figure 1, natural sites are denoted with triangles and artificial sites with filled circles. The second column of plots shows the same nMDS ordinations as the first column including the output of a generalised additive model with a 2D smoothed function for each of the significant environmental / impact variables overlaid; temperature - mean sea surface temperature $\left({ }^{\circ} \mathrm{C}\right)$; Chlorophyll $a$ - chlorophyll $a$ concentration $\left(\mathrm{mg} \mathrm{m}^{-3}\right)$; impact - human marine impact score (unitless measurement, see details in text) against the two nMDS axes. The Venn diagrams indicate the percentage total of the variance in the community dissimilarity explained by each significant variable, derived using variance partitioning of a distance-based redundancy analysis. 


\section{Distance-decay}

433 Distance-decay slopes for all observations showed an exponential decrease in compositional

434 similarity as the distance between sites increased (Fig. 3a). Regression models of $\log 10$

435 transformed compositional similarity indicated that this slope was statistically significant in all

436 cases $(\mathrm{P}<0.001$ for all taxonomic groups, full model output in Supplementary Information 5). In

437 the metazoan dataset the model showed a significant difference in the slope between artificial

438 and natural sites $\left(\mathrm{F}_{3,76}=47.73, \mathrm{p}<0.001\right)$. No statistically significant difference was found

439 between site types in the protist or bacteria data, and the same pattern was observed within

440 taxonomic groups for the metazoan and protistan subsets across both the COI and 18S data

441 (Supplementary Information 5). 
a

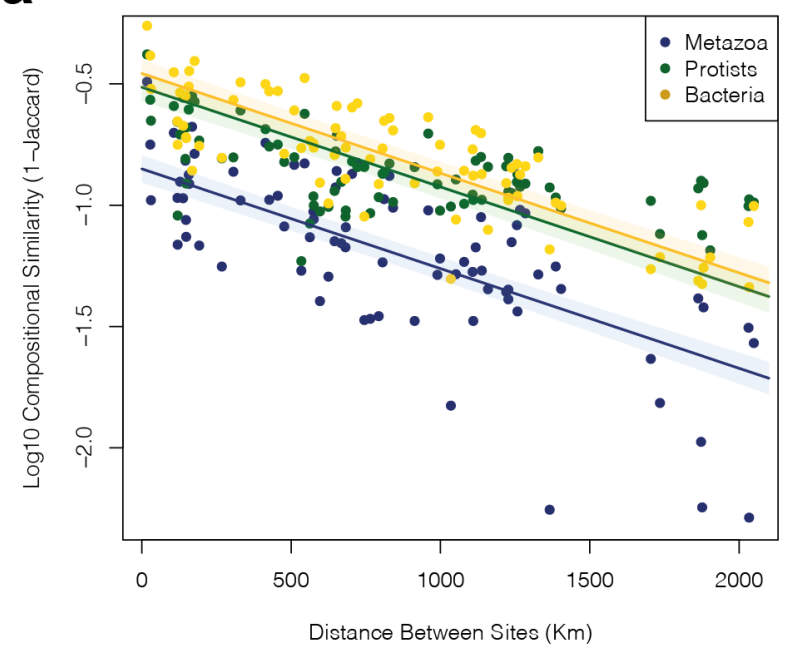

C

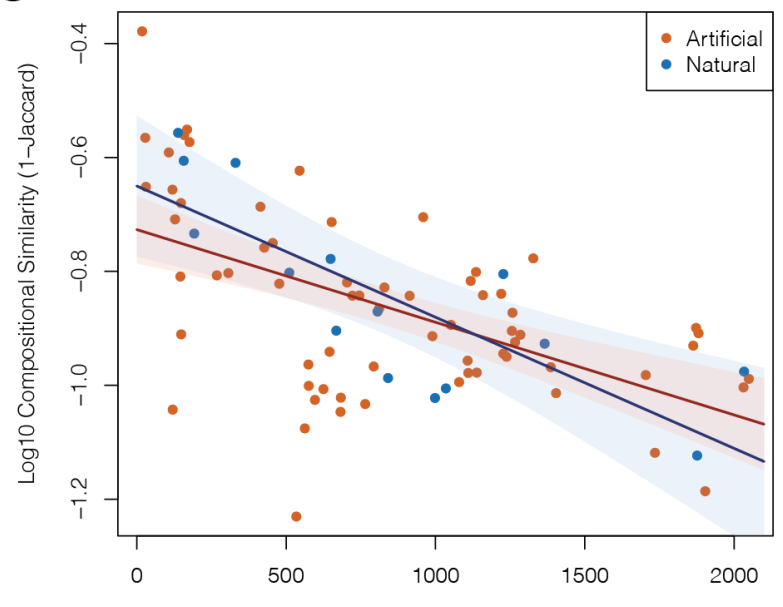

Distance Between Sites (Km) b

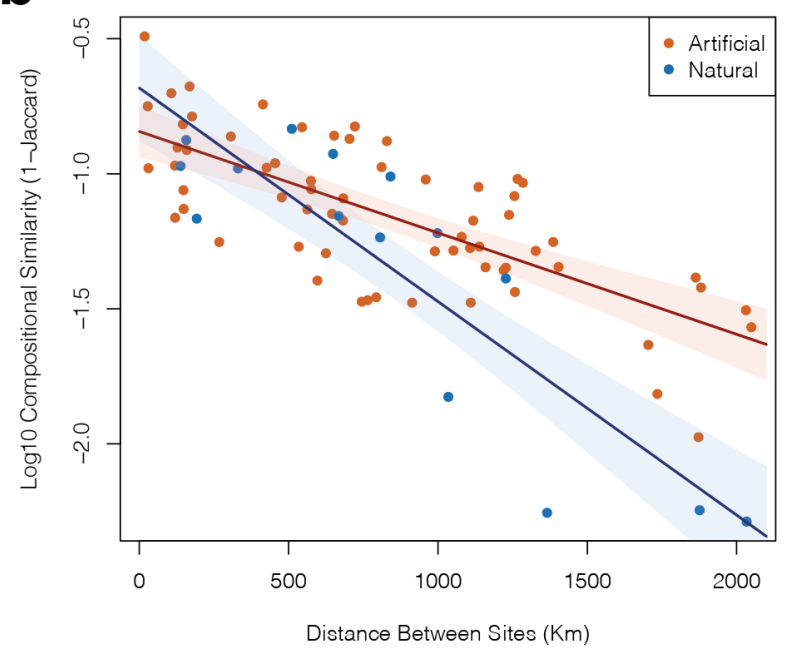

d

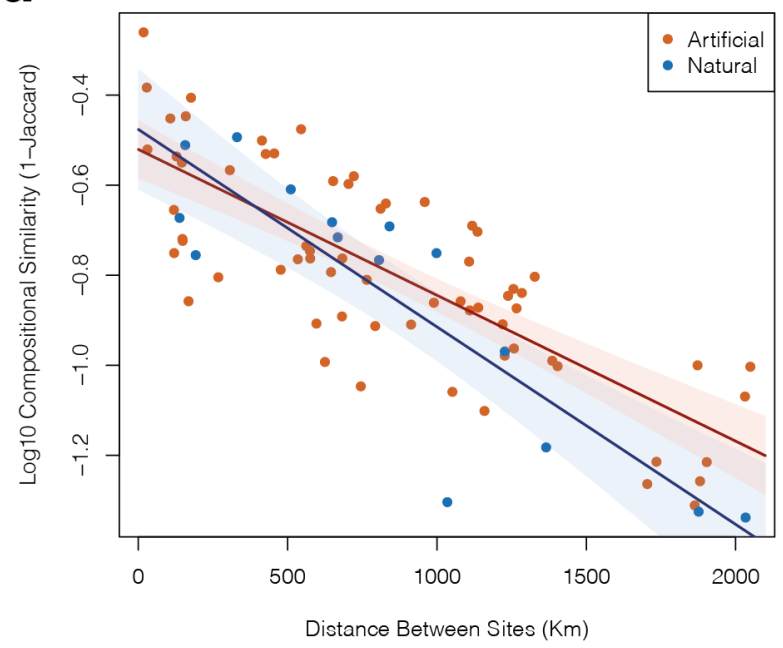

Figure 3. Plots showing distance between sites and community similarity measured using environmental

445 DNA metabarcoding across South Africa. Logarithmically (base 10) transformed compositional similarity against distance is shown in a, which includes all datasets. Comparisons between artificial (coloured in red) and natural (coloured blue) sites are shown for $\mathbf{b}$ metazoans, $\mathbf{c}$ COI protists and $\mathbf{d}$ bacteria. 95\% confidence intervals from the regression models are shown as light shaded areas around each regression slope. 


\section{Discussion}

452 Here, we showed that metazoans, protists and bacteria can have similar biogeographic patterns

453 along an extensive and heterogenous coastline. We found that these remarkably consistent

454 patterns could be partially explained by measured environmental conditions (chlorophyll $a$ and

455 temperature), and to a lesser extent, cumulative human impact. Additionally, we found evidence

456 for anthropogenically driven homogenisation of communities exclusively in metazoans.

457 Collectively, we provide evidence of congruent biogeographic boundaries across vastly different

458 forms of life, and demonstrate that underlying processes, such as anthropogenic alterations,

459 affect biogeographic patterns differentially across taxa.

461 Prokaryotes and eukaryotes diverged billions of years ago and have since evolved to inhabit a

462 vast range of ecological niches. Previous studies have shown both similar ${ }^{59,60}$ and dissimilar ${ }^{61}$

463 patterns of $\beta$ diversity between macro- and microscopic species across environmental and

464 geographic gradients. Recent work has explored biogeographic regionalisation in marine

465 plankton across kingdoms ${ }^{15}$, showing that smaller planktonic organisms such as bacteria may

466 have greater biogeographic structuring compared to larger metazoans or protists ${ }^{15}$. Together this

467 evidence suggests that different ecological processes drive a number of taxon-specific responses

468 to produce patterns that are not universal across systems at different spatial scales ${ }^{13}$. Here we

469 observed similar biogeographic patterns across kingdoms (Fig. 2), providing clear evidence of

470 cross-phyla biogeographical congruence.

472 Our analyses suggest that environmental variables such as temperature or chlorophyll $a$

473 concentration influenced the structure of marine communities across the study region (Fig. 2).

474 Global studies of biogeographic patterns have shown a central role of temperature in the

475 structuring of both microbial ${ }^{12}$ and larger planktonic life $\mathrm{e}^{15,62}$ across the ocean. There is growing

476 evidence that the range boundaries of marine organisms closely track their thermal limits 4.

477 Therefore, a general expectation was that species would remain within their thermal niche

478 resulting in temperature-structured communities as observed here. In contrast to temperature,

479 salinity had a minor role in structuring the studied communities (Fig. 2), an observation

480 previously reported for the global ocean ${ }^{12}$, with exceptions found in microbial ${ }^{63}$ and meiofaunal ${ }^{64}$

481 life in regions with unusually strong salinity gradients (e.g. Baltic Sea). The SSS range across 
482 our study system was very narrow (35.04 - 35.38ppt) and so the negligible observed effect was

483 expected. In the case of protists, biogeographic patterns showed a stronger association with

484 primary productivity (measured here as chlorophyll $a$ concentration). Previous research has

485 shown little or no role of productivity in driving coastal and oceanic scale biodiversity

486 patterns ${ }^{15,62}$. However, these studies explored the global role of various environmental variables;

487 the significance of more localised oceanographic systems such as upwelling (as along the

488 western coast of southern Africa) might not be as apparent in global analyses.

490 Anthropogenic activities are known to alter both the physico-chemical properties of the marine

491 environment and the trophic and ecological properties of ecosystems ${ }^{65}$. In our study system,

492 human impact provided some explanatory power to understand the observed community

493 structure, but to a much lesser extent compared to environmental variables (Fig. 2). The human

494 impact index used here ${ }^{51}$ covered a large number of different types of impact (e.g. pollution,

495 shipping intensity) but even this granular approach adequately explained a small proportion of

496 the total variation in ASVs observed among sampling sites. Previous work on marine metazoans

497 has shown a strong effect of proximate urbanisation ${ }^{34}$ and the ecological drivers produced

498 through anthropogenic activities are well documented ${ }^{65}$. Interestingly, the pervasive and

499 conspicuous urbanisation of the marine environment in the study area showed a much weaker

500 effect on biogeographic patterns than other explanatory variables (Fig. 2). Anthropogenic

501 pressures have become a major ecological driver only relatively recently in evolutionary time,

502 with the most dramatic changes in biodiversity occurring within the $21^{\text {st }}$ century ${ }^{3}$. It is clear that

503 human activities are altering evolutionary trajectories ${ }^{65}$, either through extinction, range

504 expansions or contractions. However, our data suggests that centuries of human impacts in our

505 study system have not yet demonstrably altered the main observed biogeographic boundaries

506 across taxa.

508 Previous work on biotic homogenisation has shown a dramatic effect on whole communities at

509 both regional ${ }^{18,66}$ and global scales ${ }^{24,25}$. Here, we found evidence for biotic homogenisation along

510 the South African coastline only in metazoan species, with a difference in the slope of a distance-

511 decay relationship between artificial and natural sites. This pattern was consistent for metazoans

512 across the gene regions considered (Supplementary Information 5), with more homogenous 
513 community composition between artificial sites. Pervasive vessel activity in the region ${ }^{31}$, along

514 with evidence that artificial environments are hotspots for metazoan invasions ${ }^{33}$, suggest that

515 introduced metazoans are contributing to homogenisation of coastal communities. Further work

516 should incorporate time series data to explore biotic homogenisation, given the significant but

517 minor role of human impact in structuring ecological communities across the region.

519 Both environmental parameters and species interactions have a clear-cut effect on marine

520 community structure across kingdoms of life ${ }^{67}$, but the comparative role of deterministic

521 (environmental filtering, niche processes, etc.) and stochastic processes (ecological drift, random

522 extinction, etc.) in explaining the observed patterns remains uncertain. The classical

523 deterministic theory (Baas Becking hypothesis ${ }^{68}$, often summarised as 'everything is

524 everywhere') of microbial biogeography postulates that due to vast population sizes and

525 dispersal, microbes are found in all environments and the variation in abiotic conditions selects

526 for those that make up the vast majority of species in each region. This theory ignores neutral

527 processes which have been shown to have a critical role in structuring microbial biogeography

528 across biomes ${ }^{69}$. In line with previous efforts studying deterministic and stochastic processes

529 across taxonomic kingdoms ${ }^{70,71}$, we found that the majority of the observed variation could not

530 be fully explained for both prokaryotic and eukaryotic species (Fig. 2). Indeed, recent

531 biogeographic research in the oceans has provided both theoretical ${ }^{72}$ and empirical ${ }^{15}$ evidence of $^{2}$

532 strong biogeographic patterns driven by both stochastic and deterministic forces, but much of the

533 observed variation between communities remains unexplained. Understanding the comparative

534 roles of different community structuring processes requires a more comprehensive examination

535 of observed variance between communities, the interactions between taxa, and the broader role

536 of the environmental conditions where they live.

538 Several recent innovations will provide valuable data to help uncover the unexplained variation

539 in community structure. For example, the extraction and analysis of sedimentary ancient DNA

540 allows the reconstruction of high-resolution biodiversity change over time ${ }^{73}$. By rewinding the

541 ecological clock, the approach provides evidence to evaluate the role of deterministic processes

542 in reconstructions of community composition through time relative to documented changes in

543 environmental proxies. In conjunction, the analysis of networks from molecular data, in 
544 conjunction with life history parameters, could facilitate the hypothesis testing of species

545 interactions that may further elucidate the role of ecological interactions (e.g. Djurhuus, et al. ${ }^{14}$ )

546 in structuring biogeographic patterns. Finally, very high-resolution multi-spectral remote sensing

547 data (e.g. WorldView-3, <100m) will provide unparalleled insights into the role of

548 environmental forces on the distribution of ecological communities ${ }^{74}$. 


\section{Acknowledgments}

551 LH acknowledges the assistance of Molly Czachur and Thomas Grevesse during field surveys,

552 Sophie von der Heyden for lab consumables and assistance with fieldwork logistics, and both

553 Jamie Hudson and Ivan Haigh for assistance with remote sensing data. LH acknowledges Shirley

554 Parker-Nance and the Elwandle Node of the South African Environmental Observation Network

555 for assistance and in-country logistics. All marina owners and operators are acknowledged for

556 field site access. We acknowledge the Environmental Sequencing Facility from the National

557 Oceanography Centre, Southampton for access and sequencing assistance. We thank the IRIDIS

558 High Performance Computing Facility, and associated support services at the University of

559 Southampton. LH was supported by the Natural Environmental Research Council (grant number

560 NE/L002531/1). The UK Research and Innovation Newton Fund (grant: ES/N013913/1)

561 supported LH's research stay in South Africa. 


\section{Data Availability}

563 Raw Illumina sequencing data are available from the European Nucleotide Archive under study

564 accession number PRJEB38452, sample specific accessions are provided in Supplementary

565 Table 10. Associated metadata, R scripts and intermediate files are available at the following

566 DOI:10.5281/zenodo.4384644.

567

\section{Code Availability}

569 All code used in the current study can be found at the following DOI:10.5281/zenodo.4384644. 
5731 Spalding, M. D. et al. Marine ecoregions of the world: A bioregionalization of coastal and shelf areas. BioScience 57, 573-583, doi:10.1641/B570707 (2007). benthic invertebrates applied to the selection of priority conservation areas. Diversity and Distributions 8, 129-145, doi:10.1046/j.1472-4642.2002.00132.x (2002).

3 Pecl, G. T. et al. Biodiversity redistribution under climate change: Impacts on ecosystems and human well-being. Science 355, doi:10.1126/science.aai9214 (2017).

5804 Sunday, J. M., Bates, A. E. \& Dulvy, N. K. Thermal tolerance and the global redistribution of animals. Nature Climate Change 2, 686-690, doi:10.1038/nclimate1539 (2012).

5 Wallace, A. R. The geographical distribution of animals, with a study of the relations of living and extinct faunas as elucidating the past changes of the earth's surface. (Macmillan and Co., 1876).

5866 Holt, B. G. et al. An update of Wallace's zoogeographic regions of the world. Science 339, 74-78, doi:10.1126/science.1228282 (2013). Ficetola, G. F., Mazel, F. \& Thuiller, W. Global determinants of zoogeographical boundaries. Nature Ecology and Evolution 1, 89, doi:10.1038/s41559-017-0089 (2017).

15 Richter, D. J. et al. Genomic evidence for global ocean plankton biogeography shaped by

16 Naeem, S., Duffy, J. E. \& Zavaleta, E. The functions of biological diversity in an age of Kocsis, A. T., Reddin, C. J. \& Kiessling, W. The stability of coastal benthic biogeography over the last 10 million years. Global Ecology and Biogeography 27, 11061120, doi:10.1111/geb.12771 (2018).

615 diversity. Proceedings of the National Academy of Sciences of the United States of America 114, 5653-5658, doi:10.1073/pnas.1702297114 (2017).

Costello, M. J. et al. Marine biogeographic realms and species endemicity. Nature Communications 8, doi:10.1038/s41467-017-01121-2 (2017).

12 Sunagawa, S. et al. Ocean plankton. Structure and function of the global ocean microbiome. Science 348, 1261359, doi:10.1126/science.1261359 (2015).

13 Shade, A. et al. Macroecology to Unite All Life, Large and Small. Trends in Ecology and Evolution 33, 731-744, doi:10.1016/j.tree.2018.08.005 (2018).

14 Djurhuus, A. et al. Environmental DNA reveals seasonal shifts and potential interactions in a marine community. Nature Communications 11, 254, doi:10.1038/s41467-01914105-1 (2020). large-scale current systems. bioRxiv, 867739, doi:10.1101/867739 (2019). extinction. Science 336, 1401-1406, doi:10.1126/science.1215855 (2012).

7 Tilman, D., Isbell, F. \& Cowles, J. M. Biodiversity and Ecosystem Functioning. Annual Review of Ecology, Evolution, and Systematics 45, 471-493, doi:10.1146/annurevecolsys-120213-091917 (2014).

18 Finderup Nielsen, T., Sand-Jensen, K., Dornelas, M. \& Bruun, H. H. More is less: net gain in species richness, but biotic homogenization over 140 years. Ecology Letters 22, 1650-1657, doi:10.1111/ele.13361 (2019). 
61719 Blowes, S. A. et al. The geography of biodiversity change in marine and terrestrial

618 assemblages. Science 366, 339-345, doi:10.1126/science.aaw1620 (2019).

$61920 \quad$ Dornelas, M. et al. Assemblage time series reveal biodiversity change but not systematic loss. Science 344, 296-299, doi:10.1126/science.1248484 (2014).

622

21 Olden, J. D. \& Rooney, T. P. On defining and quantifying biotic homogenization. Global Ecology and Biogeography 15, 113-120, doi:10.1111/j.1466-822X.2006.00214.x (2006). Stuart-Smith, R. D. et al. Integrating abundance and functional traits reveals new global hotspots of fish diversity. Nature 501, 539-542, doi:10.1038/nature12529 (2013).

23 Mouillot, D. et al. Rare species support vulnerable functions in high-diversity ecosystems. PLoS Biology 11, e1001569, doi:10.1371/journal.pbio.1001569 (2013). regions. Ecology Letters 22, 1297-1305, doi:10.1111/ele.13321 (2019). Capinha, C., Essl, F., Seebens, H., Moser, D. \& Pereira, H. M. The dispersal of alien species redefines biogeography in the Anthropocene. Science 348, 1248-1251, doi:10.1126/science.aaa8913 (2015).

Azam, F. \& Malfatti, F. Microbial structuring of marine ecosystems. Nature Reviews Microbiology 5, 782-791, doi:10.1038/nrmicro1747 (2007). Deiner, K. et al. Environmental DNA metabarcoding: Transforming how we survey animal and plant communities. Molecular Ecology 26, 5872-5895, doi:10.1111/mec.14350 (2017). zoogeographic and functional approach to the selection of marine reserves on the west coast of South Africa. South African Journal of Marine Science 12, 341-354,

29 Griffiths, C. L., Robinson, T. B., Lange, L. \& Mead, A. Marine biodiversity in South Africa: an evaluation of current states of knowledge. PLoS One 5, e12008, doi:10.1371/journal.pone.0012008 (2010).

30 Griffiths, C. L. et al. Impacts of human activities on marine animal life in the Benguela: A historical overview. Oceanography and Marine Biology: an Annual Review 42, 303392, doi:10.1201/9780203507810.ch8 (2004).

31 Kaluza, P., Kolzsch, A., Gastner, M. T. \& Blasius, B. The complex network of global cargo ship movements. Journal of The Royal Society Interface 7, 1093-1103, doi:10.1098/rsif.2009.0495 (2010).

32 Rius, M. et al. Range expansions across ecoregions: interactions of climate change, physiology and genetic diversity. Global Ecology and Biogeography 23, 76-88, doi:10.1111/geb.12105 (2014). environmental DNA metabarcoding of sediment and water. Scientific Reports 9, 11559 , doi:10.1038/s41598-019-47899-7 (2019).

34 Kelly, R. P. et al. Genetic signatures of ecological diversity along an urbanization gradient. PeerJ 4, e2444, doi:10.7717/peerj.2444 (2016).

35 Spens, J. et al. Comparison of capture and storage methods for aqueous macrobial eDNA using an optimized extraction protocol: advantage of enclosed filter. Methods in Ecology and Evolution 8, 635-645, doi:10.1111/2041-210x.12683 (2017). 
66136 Zhan, A. et al. High sensitivity of 454 pyrosequencing for detection of rare species in aquatic communities. Methods in Ecology and Evolution 4, 558-565, doi:10.1111/2041210x.12037 (2013).

66437 Leray, M. et al. A new versatile primer set targeting a short fragment of the mitochondrial COI region for metabarcoding metazoan diversity: application for characterizing coral reef fish gut contents. Frontiers in Zoology 10, 34, doi:10.1186/1742-9994-10-34 (2013).

38 Takahashi, S., Tomita, J., Nishioka, K., Hisada, T. \& Nishijima, M. Development of a prokaryotic universal primer for simultaneous analysis of Bacteria and Archaea using

\&

46 Edgar, R. C. Updating the 97\% identity threshold for 16 S ribosomal RNA OTUs. Bioinformatics 34, 2371-2375, doi:10.1093/bioinformatics/bty113 (2018). Trends in Ecology \& Evolution 35, 43-55 (2020).

49 Zweng, M. M., J. R. Reagan, D. Seidov, T. P. Boyer, R. A. Locarnini, H. E. Garcia, A. V. Mishonov, O. K. Baranova, K. Weathers, C. R. Paver, and I. Smolyar,. World Ocean Atlas 2018, Volume 2: Salinity. 50 (2019).

50 European_Space_Agency. Ocean Colour Climate Change Initiative Dataset, Version 4.2. (2020).

51 Halpern, B. S. et al. Recent pace of change in human impact on the world's ocean. Scientific Reports 9, 11609, doi:10.1038/s41598-019-47201-9 (2019).

52 Anderson, M. J. in Wiley Stats Ref: Statistics Reference Online (eds N. Balakrishnan et al.) 1-15 (John Wiley \& Sons, Ltd, 2014).

53 Oksanen, J. et al. Vegan: community ecology package. R package, Version 2.5-6 (2011). 
54 Kreft, H. \& Jetz, W. A framework for delineating biogeographical regions based on species distributions. Journal of Biogeography 37, 2029-2053, doi:10.1111/j.13652699.2010.02375.x (2010).

55 Salazar, G. EcolUtils: Utilities for community ecology analysis. R package version 0.1 (2018).

56 Anderson, M. J. Distance-Based Tests for Homogeneity of Multivariate Dispersions. Biometrics 62, 245-253, doi:10.1111/j.1541-0420.2005.00440.x (2006).

57 Crabot, J., Clappe, S., Dray, S. \& Datry, T. Testing the Mantel statistic with a spatiallyconstrained permutation procedure. Methods in Ecology and Evolution 10, 532-540, doi:10.1111/2041-210x.13141 (2019).

58 McArdle, B. H. \& Anderson, M. J. Fitting Multivariate Models To Community Data: A Comment On Distance-Based Redundancy Analysis. Ecology 82, 290-297, doi:10.1890/0012-9658(2001)082[0290:Fmmtcd]2.0.Co;2 (2001).

59 Rapacciuolo, G., Beman, J. M., Schiebelhut, L. M. \& Dawson, M. N. Microbes and macro-invertebrates show parallel $\beta$-diversity but contrasting $\alpha$-diversity patterns in a marine natural experiment. Proceedings of the Royal Society B: Biological Sciences 286, 20190999, doi:doi:10.1098/rspb.2019.0999 (2019).

60 Astorga, A. et al. Distance decay of similarity in freshwater communities: do macro- and microorganisms follow the same rules? Global Ecology and Biogeography 21, 365-375, doi:10.1111/j.1466-8238.2011.00681.x (2012).

61 Wang, J. et al. Patterns of elevational beta diversity in micro- and macroorganisms. Global Ecology and Biogeography 21, 743-750, doi:10.1111/j.1466-8238.2011.00718.x (2012).

62 Tittensor, D. P. et al. Global patterns and predictors of marine biodiversity across taxa. Nature 466, 1098-U1107, doi:10.1038/nature09329 (2010).

63 Herlemann, D. P. et al. Transitions in bacterial communities along the $2000 \mathrm{~km}$ salinity gradient of the Baltic Sea. ISME Journal 5, 1571-1579, doi:10.1038/ismej.2011.41 (2011).

64 Broman, E. et al. Salinity drives meiofaunal community structure dynamics across the Baltic ecosystem. Molecular Ecology 28, 3813-3829, doi:10.1111/mec.15179 (2019).

65 Shochat, E., Warren, P. S., Faeth, S. H., McIntyre, N. E. \& Hope, D. From patterns to emerging processes in mechanistic urban ecology. Trends in Ecology and Evolution 21, 186-191, doi:10.1016/j.tree.2005.11.019 (2006).

66 Blouin, D., Pellerin, S. \& Poulin, M. Increase in non-native species richness leads to biotic homogenization in vacant lots of a highly urbanized landscape. Urban Ecosystems 22, 879-892, doi:10.1007/s11252-019-00863-9 (2019).

67 Lima-Mendez, G. et al. Determinants of community structure in the global plankton interactome. Science 348, 1262073, doi:10.1126/science.1262073 (2015).

68 Baas-Becking, L. G. M. Geobiologie; of inleiding tot de milieukunde. (WP Van Stockum \& Zoon NV, 1934).

69 Hanson, C. A., Fuhrman, J. A., Horner-Devine, M. C. \& Martiny, J. B. Beyond biogeographic patterns: processes shaping the microbial landscape. Nature Reviews Microbiology 10, 497-506, doi:10.1038/nrmicro2795 (2012).

70 Farjalla, V. F. et al. Ecological determinism increases with organism size. Ecology 93, 1752-1759, doi:10.1890/11-1144.1 (2012). 
$75071 \quad \mathrm{Wu}, \mathrm{W}$. X. et al. Contrasting the relative importance of species sorting and dispersal

751 limitation in shaping marine bacterial versus protist communities. ISME Journal 12, 485-

752 494, doi:10.1038/ismej.2017.183 (2018).

75372 Hellweger, F. L., van Sebille, E. \& Fredrick, N. D. Biogeographic patterns in ocean

754 microbes emerge in a neutral agent-based model. Science 345, 1346-1349,

755 doi:10.1126/science.1254421 (2014).

75673 Balint, M. et al. Environmental DNA time series in ecology. Trends in Ecology and

$757 \quad$ Evolution 33, 945-957, doi:10.1016/j.tree.2018.09.003 (2018).

75874 He, K. S. et al. Will remote sensing shape the next generation of species distribution models? Remote Sensing in Ecology and Conservation 1, 4-18, doi:10.1002/rse2.7 